\title{
Induced mild hypothermia in children
}

\author{
RIMANTAS KEVALAS - DOVILE GRINKEVICIUTE \\ VAIDOTAS GURSKIS • ALGIMANTAS MATUKEVIČIUS \\ VYTAUTAS RAGAIŠIS • VIDMANTAS BARAUSKAS
}

RIMANTAS KEVALAS $(\bowtie) \cdot$ DOVILE GRINKEVICIUTE • VAIDOTAS GURSKIS

Department of Pediatric Diseases Lithuanian University of Health Sciences Eiveniu 2, Kaunas 50009, Lithuania Phone: + 37037326963 Fax: +37037327160 E-mail: rimantas_kevalas@hotmail.com

ALGIMANTAS MATUKEVIČIUS • VYTAUTAS RAGAIŠIS

Department of Neurosurgery Lithuanian University of Health Sciences

Kaunas, Lithuania

VIDMANTAS BARAUSKAS Department of Pediatric Surgery Lithuanian University of Health Sciences Kaunas, Lithuania

\begin{abstract}
The objective of this study was to measure outcomes and to determine the safety and effectiveness of mild induced hypothermia in children after traumatic and posthypoxic brain injury.

Methods. Forty patients, following traumatic or posthypoxic brain injury, were involved in the study. Mean age was $10.7 \pm$ 0.8 years. Median GCS (Glasgow Coma Scale) was 6.0 (4-7) and mean PIM2 (Pediatric Index of Mortality) $14.6 \pm 3.8 \%$. Results. GOS (Glasgow Outcome Scale) of 5 was assigned for 15 (37.5\%) patients, GOS 4 for 14 (35.0\%), GOS 3 for 7 (17.5\%) and GOS 2 for 4 (10\%) patients. The average GOS in patients after severe head trauma was $3.6 \pm 0.9$ points and in patients with posthypoxic brain injury 5 points, $(p<0.05)$. No life threatening complications occurred.

Conclusion. Mild induced hypothermia can be safely used in pediatric patents after severe traumatic or posthypoxic brain injury. This method may be of benefit while improving outcomes in children.
\end{abstract}

Key words: traumatic brain injury, posthypoxic brain injury, children, hypothermia, outcome, Pediatric Index of Mortality

\section{Introduction}

Brain injury is a significant cause of death and disability in children. At least one third of survivors sustain moderate to severe neurological sequelae. Induced hypothermia can be used to protect the brain from post-ischemic and traumatic neuronal injury. Neurological impairment depends not only on primary, but also on secondary brain injury. In case of strangulation or neardrowning, primary brain injury is initial- ly associated with tissue hypoxia and ischemia, secondary injury occurs from reperfusion, sustained acidosis, cerebral edema, hyperglycemia, release of excitatory neurotransmitters, seizures, hypotension and impaired cerebral autoregulation. In trauma cases, primary brain injury occurs at the time of impact, either by a direct injury to the brain parenchyma or by an injury to the long white matter tracts through acceleration - deceleration forces. (1) Secondary injury is represented by systemic and intracranial events that occur in response to the primary injury and further contribute to neuronal damage and cell death. The systemic events are hypotension, hypoxia and hypercapnia. The intracranial events are a series of inflammatory changes and pathophysiological perturbations that occur immediately after the primary injury and continue over time. It remains a challenge to develop therapies that target multiple injury cascades to successfully improve outcome for a heterogeneous brain injury group. One highly promising therapy that targets multiple pathological mechanisms caused by traumatic or posthypoxic brain injury is mild induced hypothermia (MIH). The use of cold as a therapeutic agent has received renewed interest in the last decade though it has a long and 
colorful history reaching back to 3500 B.C., when Edwin Smith Papyrus, the most ancient medical text known, made numerous references to the use of cold as a therapy. (2) In the 18th century surgeon-in-chief of the Napoleonic armies, Baron Dominique Jean Larrey, observed that severely wounded soldiers lying closer to the campfires died at a higher rate than those in more remote, colder areas. (3) Intentional cooling to induce a state of hypothermia was described as clinical therapy for malignancies and head injuries by Temple Fay in 1938. $(2,4)$ Since the 1950s, induced hypothermia has been used in an attempt to attenuate secondary brain injury following a variety of primary insults including carbon monoxide poisoning, status epilepticus, ischemic stroke and more recently for patients with hepatic encephalopathy.(2)

The mechanism by which hypothermia conveys its neuroprotective effect is unclear. We know that hypothermia reduces global cerebral metabolism, cerebral oxygen demands, lactic acid accumulation, calcium influx in neurocytes, free radicals production, lipid peroxidation, posttraumatic level of excitatory neurotransmitter, inhibition of apoptosis, and lowers the damage of cytoskeletal structure. (5)

There is clear evidence of a beneficial effect of induced hypothermia on neurological outcome in patients following cardiac arrest $(6,7)$ or in neonates with hypoxic - ischemic encephalopathy, (8) but things in traumatic brain injury are controversial. (9)

In our study we aimed to determine the safety and effectiveness of $\mathrm{MIH}$ for pediatric patients after traumatic or posthypoxic brain injury.

\section{Material and methods Setting}

This prospective, observational, clinical trial was conducted at the Hospital of Lithuanian University of Health Sciences (HLUHS), Department of Pediatric Diseases, Kaunas, Lithuania. The study took place in the Pediatric Intensive Care Unit (PICU) - an eight bed unit admitting, on a non - elective basis, approximately
1000 children per year and providing the services of a pediatric intensive care physician, pediatric neurosurgeon, pediatric surgeon, pediatric neurologist and radiologist 24 hours a day.

\section{Patients}

All pediatric patients treated with mild induced hypothermia at the PICU of HLUHS, after severe traumatic or posthypoxic brain injury, during a 60 - month period (January 1, 2005, to January 1, 2010), were enrolled. Inclusion criteria were: post-resuscitation Glasgow Coma Scale (GCS) $\leq 8$, or rapid deterioration of consciousness (2 or more points of GCS in one hour) and ability to start the hypothermia protocol in the first six hours after the event. Patients were divided into two groups according to the type of injury (traumatic or posthypoxic).

\section{Data collection}

In - hospital variables (age, sex, diagnosis, length of PICU stay and artificial ventilation) were recorded in the patient's hospital chart and in an electronic database. Temperature, heart rate, mean arterial pressure, urine output, volumes and types of intravenous fluids administered were recorded hourly on patients' care sheets. Laboratory values (serum sodium, potassium, glucose, lactate level, blood gases, coagulation indicators) were analyzed every four hours. C reactive protein and blood count were performed daily. Hypothermia induced complications were recorded in the patient's hospital chart at the moment of their occurrence. GCS and Pediatric Index of Mortality (PIM2) were calculated within the first hour of admission. Calculation of GCS was based on eye opening, motor response and verbal response; calculation of PIM2 was calculated from the eight variables recorded at the first hour of admission to the PICU. Rectal temperature was measured continuously electronically starting with temperature on arrival to temperature at the end of rewarming.

\section{Patient care}

All patients were artificially ventilated and received analgesia through continuous intravenous morphine infusion, sedation with benzodiazepines and muscle relaxation intravenously. Intracranial pressure (ICP) was measured only in patients after traumatic brain injury. All patients after traumatic brain injury were treated according to severe head injury treatment protocol based on ICP and cerebral perfusion pressure (CPP) targeted therapy.

Nutritional support by the enteral route was started during the rewarming period. Cooling procedures included surface cooling techniques (cooling blankets, ice pads and cool pads), gastric lavage with iced fluid, external ventilator for body surface cooling and use of room temperature air in the ventilation circuit. The cooling protocol was commenced within six hours of the event. The aim of the induction phase was to reach 33-34 ${ }^{\circ} \mathrm{C}$ core temperature within 2 3 hours. The aim of the maintenance phase was to maintain core temperature at $33-34^{\circ} \mathrm{C}$, to maintain hemodynamic stability and normal physiological and laboratory parameters. The duration of this phase according to our protocol was 48 hours for traumatic brain injury and 24 hours for posthypoxic brain injury. The aim of the rewarming phase was to achieve normal body temperature $\left(36-37^{\circ} \mathrm{C}\right)$. Passive rewarming was used. The rewarming rate had to be no faster than $1^{\circ} \mathrm{C}$ per four - hour period.

\section{Measuring of outcome}

Outcome measure was the assessment of patients according to the five - category Glasgow Outcome Scale (GOS), which was conducted on discharge from hospital. GOS of 1 was applied to patients who died, GOS of 2 - for those who appeared to be in a vegetative state (unresponsive, unable to interact with the environment), GOS of 3 - for severe disability (able to follow commands, unable to live independently), GOS of 4 - for moderate disability (able to live independently, unable to return to work or school), GOS of 5 - for full recovery (able to return to work or school). Outcome was assessed on patient's discharge from the hospital. 


\section{Statistical analysis}

Statistical analysis was performed with SPSS 13 for Windows. Quantitative variables were expressed as mean \pm standard deviation or median (interquartile range) as appropriate. Qualitative variables were expressed as numbers and percentages. For comparing distribution of variables in two Mann - Whitney $\cup$ test was used. Statistical significance was taken as $p<0.05$.

\section{Results Patient's characteristics}

Mild hypothermia was used in 43 cases during the research period. Three patients were excluded from the study; one patient was excluded because of misdiagnosis of encephalitis, one patient had congenital cerebral dysplasia and severe psychomotor retardation, and one patient died on the first day of admission from severe lung injury (major aspiration of oilseed rape). The study involved 40 patients: 27 (67.5\%) boys and 13 (32.53\%) girls. Mean age was $10.7 \pm 0.8$ years, median weight 39.6 (12 - 70) kg, median GCS 6.0 (4 $-7)$ and mean PIM2 $14.6 \pm 3.8 \%$. The origin of brain injury was trauma in 30 cases (pedestrian - 10 cases; passenger - 16 cases; falls -4 cases) and posthypoxic brain injury in 10 cases (submersion - 4 cases; strangulation 5 cases, one patient was buried under the snow). We had no patients with cardiac rhythm disturbances in our study. Baseline characteristics of the patients, according to the type of brain injury, are shown in table 1. Demographic data were similar in both groups, but PIM 2 was significantly higher in the posthypoxic group.

\section{Patient management and length of stay}

All patients were managed according to strict protocols: cooling protocol for all patients plus ICP targeted severe head trauma protocol for head trauma patients. Normovolaemia was maintained in all patients. Thirty three patients required vasoactive agents for achieving hemodynamic stability. Dopamine was required in 24 (60.0\%) cases

Table 1. Baseline characteristics of the groups.

\begin{tabular}{llll}
\hline Characteristic & $\begin{array}{l}\text { Traumatic brain } \\
\text { injury group } \\
(\mathrm{N}=30)\end{array}$ & $\begin{array}{l}\text { Posthypoxic } \\
\text { brain injury } \\
\text { group } \\
(\mathrm{N}=10)\end{array}$ & p value \\
\hline Age (years)* & $11.0 \pm 0.8$ & $10.1 \pm 1.8$ & $>0.05$ \\
\hline Male sex - no. (\%) & $20(66.7)$ & $7(70.0)$ & $>0.05$ \\
\hline Weight - kg (IQR) & $40.0(20.0-60.0)$ & $34.0(15.0-55.0)$ & $>0.05$ \\
\hline $\begin{array}{l}\text { GCS on admission }- \\
\text { median (IQR) }\end{array}$ & $6(4.7-8.0)$ & $4.5(3.0-6.2)$ & $>0.05$ \\
\hline PIM2 on admission* & $5.9 \pm 0.4$ & $40.3 \pm 12.0$ & $<0.05$ \\
\hline
\end{tabular}

*Means $\pm \mathrm{SD}$

GCS, Glasgow Coma Scale; IQR, interquartile range; PIM2, Pediatric Index of Mortality.

Table 2. Duration of mechanical ventilation and length-of-stay in PICU and HLUHS according to type of injury.

\begin{tabular}{llll}
\hline Variable & $\begin{array}{l}\text { Traumatic brain } \\
\text { injury }\end{array}$ & $\begin{array}{l}\text { Posthypoxic } \\
\text { brain injury }\end{array}$ & p value \\
\hline $\begin{array}{l}\text { Mechanical ventila- } \\
\text { tion (days) }\end{array}$ & $8.9 \pm 5.5$ & $3.2 \pm 2.0$ & $<0.05$ \\
\hline $\begin{array}{l}\text { Length-of-stay in } \\
\text { PICU (days) }\end{array}$ & $13.0 \pm 7.8$ & $4.7 \pm 2.7$ & $<0.05$ \\
\hline $\begin{array}{l}\text { Length-of-stay in } \\
\text { HLUHS (days) }\end{array}$ & $42.4 \pm 23.9$ & $14.0 \pm 9.9$ & $<0.05$ \\
\hline
\end{tabular}

Values are expressed as means $\pm \mathrm{SD}$

HLUHS, Hospital of Lithuanian University of Health Sciences; PICU, Pediatric Intensive Care Unit.

and two vasoactive agents - dopamine + norepinephrine - were required in $9(22,5 \%)$ cases. In $7(17.5 \%)$ cases vasopressors were not administered. Distribution of vasopressor requirements according to the type of head injury is shown in figure 1 . Enteral nutrition was administered to all patients. No parenteral nutrition was used.

Mean length of stay in PICU was $10.9 \pm$ 7.7 days, whereas total length of hospital stay was $34.9 \pm 24.5$ days. The mean duration of mechanical ventilation was $7.5 \pm 5.4$ days. The distribution of ventilation time and length-of-stay in PICU and UHLUHS according to the type of injury is shown in table 2. The duration of mechanical ventilation, length-ofstay in PICU and total length-of-stay was significantly longer in patients with traumatic brain injury as compared with posthypoxic patients.

\section{Outcome analysis}

Full recovery (GOS 5) and moderate disability (GOS 4) that are designated as a favorable outcome were present in $29(72.5 \%)$ cases: GOS 5 was assigned to $14(35.0 \%)$ patients and GOS 4 to $15(37.5 \%)$ patients. Seven patients (17.5\%) appeared to have severe disability and $4(10.0 \%)$ a persistent vegetative state. Average GOS in patients after severe head trauma was $3.6 \pm 0.9$ 


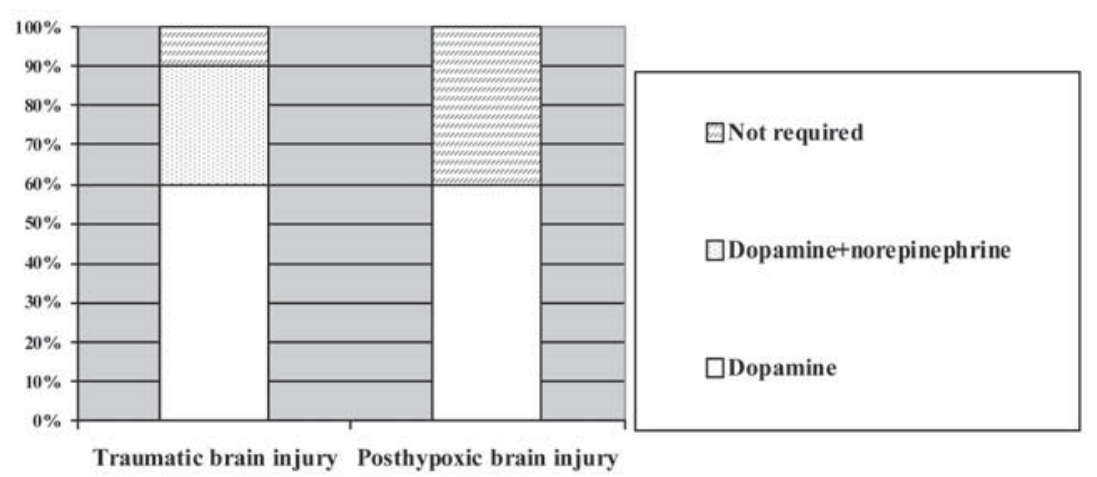

$p<0.05$

Figure 1. Requirements of vasoactive drugs according to the type of head injury.

points and in patients with posthypoxic brain injury 5 points, $(p<0.05)$. All patients after posthypoxic brain injury fully recovered. The distribution of GOS in patients after traumatic brain injury is shown in figure 2 .

\section{Safety}

A lot of medical problems occurred in the MIH patients. The most frequent problem was airways infection. It was ascertained in 29 (72.5\%) patients and occurred mostly on the second day of ventilation. Sepsis was diagnosed in $3(7.5 \%)$ patients, but it was confirmed by blood culture in only one case. Hypokalemia, defined as a serum potassium level lower than 3.8 $\mathrm{mmol} / \mathrm{l}$, was observed in 32 (80.06\%) patients and ranged from $2.4 \mathrm{mmol} / \mathrm{l}$ to $3.5 \mathrm{mmol} / \mathrm{l}$. No life threatening complications occurred due to hypokalemia and it was easily correctable. Other life threatening complications such as arrhythmia, pancreatitis, coagulative or renal disorders were not observed.

\section{Discussion}

Our results show that $\mathrm{MIH}$ in pediatric patients after posthypoxic brain injury resulted in a favorable outcome. A favorable outcome in posttraumatic brain injury was seen in $65 \%$ of trauma patients. Hypothermia did not cause any life threatening complications in both groups. The rate of patients with airway
(11) Children suffer primary traumatic brain injury similar to that in adults, but approximately $50 \%$ of children will develop diffuse cerebral swelling in the early hours after trauma. (12) In 2002 , two clinical trials proved that implementation of therapeutic hypothermia for survival in adult patients after out - of - hospital cardiac arrest results in good neurological outcome. $(6,7)$ As we know, cardiac arrest due to cardiac rhythm disturbances in children is very rare. Accidents such as trauma, strangulation and near drowning are more frequent reasons for secondary brain injury in children, resulting in poor neurologic outcome. Some studies in adults showed that therapeutic hypothermia may reduce the risks of mortality and poor neurologic outcome in adults with traumatic brain injury, $(13,14)$ but the authors state, that the evidence was not sufficient to recommend routine use of therapeutic hypothermia for traumatic brain injury outside of research settings. (15) Hypothermia affects many intracellular processes. Some of these are directly related to its neuroprotective effects. Studies show, that hypothermia tends to decrease protein oxidation and attenuates oxidative stress after severe traumatic brain injury in infants and children. (16) But induction of hypothermia causes a large number of physiological changes in the circulatory and respiratory systems, coagulation system, drug metabolism, (17) thus it can increase bleeding time due to its effect on platelet count, increase risk of arrhythmias as well as impair immune function. The physiological and pathophysiological effects of cooling largely depend on the degree of hypothermia. For example, a significant risk for severe arrhythmias occurs only at temperatures below 28 $30^{\circ} \mathrm{C}$ and is very low during mild or moderate hypothermia. Bourdages $\mathrm{M}$ et al. showed that hypothermic patients have lower heart rates than normothermic patients, but none had severe bradycardia. The most frequent arrhythmias were isolated premature atrial contractions. Most arrhythmias were of short duration and did not alter hemodynamic status. Additionally, the difference in 
the incidence of arrhythmias between the hypothermic and normothermic group was not statistically significant. (18) We did not record any arrhythmias, due to cooling itself or electrolyte disturbances, in our study and we had no bleeding or any platelet count decrease in our patients. Hypothermia, therapeutic or accidental, is usually accompanied by electrolyte alterations, especially hypokalemia. The plasma potassium level corresponds to changes in plasma phosphate $(P)$ level and that may have a role in the physiological derangements that occur during moderate hypothermia, because $\mathrm{P}$ is important for energy metabolism or blood pressure regulation. (19) Arrhythmias may be the result of hypomagnesaemia, induced by cooling patients, that's why prophylactic electrolyte supplementation should be considered and electrolyte levels should be monitored frequently. (20) Serum electrolytes were measured every four hours according to our protocol. Though hypokalemia was observed in $80 \%$ of patients, it was easily correctable and no life threatening complications occurred.

Studies show, that mild induced hypothermia is likely a safe therapeutic intervention for children after severe traumatic brain injury up to 24 hours after injury. (21) In our study we provided cooling for 48 hours in trauma patients and 24 hours in posthypoxic patients. After a pretrial study (22) in which the ability of the investigators to adhere to the clinical protocols of care, including the cooling and rewarming procedures as well as management guidelines in pediatric patients with severe traumatic brain injury, was evaluated, a multicenter, international trial of 225 children after severe traumatic brain injury was initiated. The results of this study show, that in children with severe traumatic brain injury, hypothermia therapy that is initiated within 8 hours after injury and continued for 24 hours does not improve neurologic outcome and may increase mortality. (9) One can ask whether hypothermia does more harm than good in children after severe traumatic brain injury. However, there is a big difference in the mechanism of brain injury after trauma or hypoxia. Peak neuronal death occurs days after a hypoxic episode, but hours after traumatic brain injury, (13) so the neuroprotective effect of hypothermia in case of traumatic brain injury depends on the time at which the cooling protocol is started and the aimed core temperature reached as well as on the duration of treatment and the rewarming speed. $(15,23)$ In Hutchinson's study, core temperature at a mean of $32.5^{\circ} \mathrm{C}$ was maintained for 24 hours. It is possible, that starting the cooling protocol earlier, maintaining the aimed temperature for longer and rewarming at a slower rate may have improved outcomes. Recent studies show that even pre-hospital induction of therapeutic hypothermia using infusions of $4^{\circ} \mathrm{C}$ normal saline during advanced life support is feasible, effective and safe. $(24,25)$ Some authors are raising the question of local cooling of the traumatized brain, (26) which is quite effective in neonates, (27) but is not as effective in adults and are is not available for general clinical use. (26) Our study suggests that it may be beneficial starting the cooling protocol within six hours and maintaining $\mathrm{MIH}$ protocol for 48 hours in traumatic brain injury patients and for 24 hours in posthypoxic brain injury patients. We think that longer periods of cooling do not cause more complications if the protocol is used with great attention to the prevention of side effects. Continuous hemodynamic and intracranial pressure monitoring in head trauma patients and frequent monitoring of electrolyte levels are essential.

There are no randomized trials concerning the efficacy of induced hypothermia after posthypoxic brain injury in children.
It is proven that induced hypothermia reduces the risk of death and disability in neonates with moderate or severe hypoxic - ischemic encephalopathy, (8) and there are some case reports about improved neurological outcome while using induced hypothermia in children who appeared to be near drowning victims. (28) In our study, children after posthypoxic injury had significantly worse admission scores, but their outcome was significantly better (all five children had GOS of 5 on discharge) than children with severe head trauma. One of the points to note is that in two cases of submersion cold water, the children's body temperature was lowered so we can say that cooling began at the time of the accident. On the other hand, posthypoxic brain injury has other pathophysiological mechanisms compared to severe head trauma, and that also affects outcomes.

In conclusion we want to state, that $\mathrm{MIH}$ is a safe and feasible treatment in children with traumatic or posthypoxic brain injury and it has a potential benefit on outcomes. Further studies are required for determining the optimal time of onset, duration and temperature of hypothermia, as well as for producing proper protocols for its management, because sometimes potentially beneficial treatments are under used for various reasons, such as the withdrawal of active treatment due to poor prognosis, a long delay in reaching target temperature, or thinking, that it will prolong the intensive care unit (ICU) stay and thus cause a potential burden on resources. (29)

\section{Conclusions}

Efforts to improve outcomes in children after brain injury are extremely important. Mild induced hypothermia can be safely used in pediatric patents after severe traumatic or posthypoxic brain injury. This method may be of benefit while improving outcomes in children. 


\section{REFERENCES}

1. Verive, MJ. Near Drowning [Internet]. 2009 [updated 2009 Apr 29]. Available from: http://www.scribd.com/doc/48799917/Near-DrowningeMedicine-Specialties

2. Wang H, Olivero W, Wang D, Lanzino G. Cold as a therapeutic agent. Acta Neurochir 2006;148:565-70.

3. Foster K, Stocker C, Schibler A. Controversies of prophylactic hypothermia and the emerging use of brain tissue oxygen tension monitoring and decompressive craniectomy in traumatic brain - injured children. Aust Crit Care 2010;23:4-11.

4. Vincent A. Therapeutic hypothermia for brain injury: the clinical evidence. Anaesthesia 2004;6(3):116-21.

5. Sookplung P, Vavilala MS. What is new in pediatric traumatic brain injury? Curr Opin Anaesthesiol 2009;22:572-8.

6. Bernard SA, Gray TW, Buist MD, Jones BM, Silvester W, Gutteridge G, et al. Treatment of comatose survivors of out - of - hospital cardiac arrest with induced hypothermia. N Engl J Med 2002;346(8):557-63.

7. Holzer M. Mild therapeutic hypothermia to improve the neurologic outcome after cardiac arrest. N Engl J Med 2002;346(8):549-56.

8. Shankaran S, Laptook AR, Ehrenkranz RA, Tyson JE, McDonald SA, Donovan ER, et al. Whole - body hypothermia for neonates with hypoxic - ischemic encephalopathy. N Engl J Med 2005;535:1574-84.

9. Hutchinson JS, Ward RE, Lacroix J, Hebert PC, Barnes M, Bohn DJ, et al. Hypothermia therapy after traumatic brain injury in children. N Engl J Med 2008;358(23):2447-56.

10. Berger RP, Adelson PD, Richichi R, Kochanek PM. Serum biomarkers after traumatic and hypoxemic brain injuries: insight into the biochemical response of the pediatric brain to inflicted brain injury. Dev Neurosci 2006;28:327-35.

11. Kochanek PM. The brain, the heart, and therapeutic hypothermia. Cleve Clin J Med 2009;76(2):8-12.

12. Adelson PD. Hypothermia following pediatric traumatic brain injury. J Neurotrauma 2009;26:429-36.

13. Marion DW, Penrod LE, Kelsey SF, Obrist WD, Kochanek PM, Palmer AM, et al. Treatment of traumatic brain injury with moderate hypothermia. N Engl J Med 1997;336(8):540-6.

14. Polderman KH, Joe RTT, Peederman SM, Vandertop WP, Girbes ARJ. Effects of therapeutic hypothermia on intracranial pressure and outcome in patients with severe head injury. Intensive Care Med 2002;28:1563-73.

15. Mclntyre LA, Fergusson DA, Hebert PC, Moher D, Hutchinson JS. Prolonged therapeutic hypothermia after traumatic brain injury in adults. JAMA 2003;289(22):2992-9.

16. Bayir H, Adelson PD, Wisniewski SR, Shore P, Lai MY, Brown D, et al. Therapeutic hypothermia preserves antioxidant defenses after severe traumatic brain injury in infants and children. Crit Care Med 2009;37(2):689-95.

17. Polderman KH. Application of therapeutic hypothermia in the intensive care unit. Opportunities and pitfalls of promising treatment modality - Part 2: Practical aspects and side effects. Intensive Care Med 2004;30:757-69.

18. Bourdages M, Bigras JL, Farrel CA, Hutchinson J, Lacroix J. Cardiac arrhythmias associated with severe traumatic brain injury and hypothermia therapy. Pediatr Crit Care 2010;11(3):408-14.

19. Aibiki M, Kawaguchi S, Maekawa N. Reversible hypophosphatemia during moderate hypothermia therapy for brain - injured patients. Crit Care Med 2001;29(9):1726-30.

20. Polderman KH, Peederman SM, Girbes ARJ. Hypophosphatemia and hypomagnesemia induced by cooling in patients with severe head injury. J Neurosurg 2001;94(5):697-705.

21. Adelson PD, Ragheb J, Kaney P, Brockmeyer D, Beers SR, Brown SD, et al. Phase Il clinical trial of moderate hypothermia after severe traumatic brain injury in children. Neurosurgery 2005;56(4):740-54

22. Hutchinson J, Ward R, Lacroix J, Hebert P, Skippen P, Barnes M, et al. Hypothermia pediatric head injury trial: the value of a pretrial clinical evaluation phase. Dev Neurosci 2006;28(4-5):291-301.

23. Polderman KH. Induced hypothermia and fever control for prevention and treatment of neurological injuries. Lancet 2008;371:1955-69.

24. Bruel C, Parienti JJ, Marie W, Arrot X, Daubin C, Du Cheiron D, et al. Mild hypothermia during advanced life support: a preliminary study in out - of - hospital cardiac arrest. Crit Care 2008;12(1):R31(doi:10.1186/cc6809).

25. Kim F, Olsufka M, Longstreth WTMJC, Carlbom D, Deem S, Kudenchuk P. Pilot randomized clinical trial of prehospital induction of mild hypothermia in out-of-hospital cardiac arrest patients with a rapid infusion of 4oC normal saline. Circulation 2007;115:3064-70.

26. Grande PO, Reinstrup P, Romner B. Active cooling in traumatic brain - injured patients: a questionable therapy? Acta Anesthesiol Scand 2009;53:1233-8.

27. Gluckman PD, Wyatt JS, Azzopardi D, ballard R, Edwards AD, Ferriero DM. Selective head cooling with mild systemic hypothermia after neonatal encephalopathy: multicentre randomized trial. Lancet 2005;365:663-70.

28. Hein OV, Triltsch A, Buch C, Kox WJ, Spies C. Mild hypothermia after near drowning twin toddlers. Crit Care 2004;8(5):R353-R357.

29. Hay AW, Swann DG, Bell K, Walsh TS, Cook B. Therapeutic hypothermia in comatose patients after out - of hospital cardiac arrest. Anaesthesia 2008;63:15-9. 